\title{
New technique for endoscopically-assisted particulate graft reconstruction of the mandible
}

Carlo Ferretti ${ }^{\mathrm{a}^{*}}$ (BDS, MDent, FCD(SA)MFOS), Johan Reyneke ${ }^{\mathrm{b}}$ (BChD, MChD, FCMFOS (SA), PhD), Manolis Heliotis ${ }^{c}$ (FRCS, FDSRCS, MSc, BDS, MBChB), Ugo Ripamonti ${ }^{\mathrm{d}}$ (MD, PhD)

${ }^{\text {a }}$ Private practice, Johannesburg South Africa

Formerly School of Oral Health Sciences

University of the Witwatersrand

Johannesburg, South Africa

${ }^{\mathrm{b}}$ Professor

Johan P. Reyneke

Department of Maxillofacial and Oral Surgery

University of the Western Cape, Cape Town, South Africa.

Department of Oral and Maxillofacial Surgery,

University of Oklahoma, College of Dentistry, Oklahoma City, Oklahoma.

${ }^{\mathrm{c}}$ Divisional Clinical Director

Northwick Park Hospital, London North West University Hospital

London, United Kingdom

d Professor \& Head

Bone Research Laboratory, School of Oral Health Sciences, Faculty of Health Sciences

University of the Witwatersrand, Johannesburg, South Africa

*Corresponding author: Carlo Ferretti

ferretti@mweb.co.za

Tel. 0027116159595

Fax. 0027116168649

P.O Box 75471

Gardenview

2047

SOUTH AFRICA 


\begin{abstract}
Particulate graft reconstruction of mandibular defects is a well proven technique that yields excellent restitution of mandibular osseous anatomy. If an intermediate spacer is placed at resection surgery, secondary osseous reconstruction can be accomplished with reduced access incisions and endoscopic assistance. A new technique has evolved as a result of the use of intermediate spacers following mandibular tumour resection. Two patients required resection of mandibular ameloblastoma and were reconstructed with a modified protocol with particulate grafts implanted following removal of the spacer and recipient site preparation with endoscopic visualization via small extraoral incisions. The grafts healed uneventfully and matured into ossicles suitable for the placement of osseointegrated implants.
\end{abstract}

Key word: mandible reconstruction, endoscopic 


\section{Introduction}

Grafts of particulate cancellous bone and marrow graft are well known to yield unmatched restitution of bone with low morbidity in reconstruction of the mandible. ${ }^{1-5}$ he use of an intermediate spacer (if secondary mandibular reconstruction is planned), reduces the amount of dissection required to prepare a recipient bed in which to implant a graft. It also improves the final volume of the ossicle by creating a space for implantation in which the wound has already contracted. ${ }^{6}$ While mandibular reconstruction requires extraoral exposure of the recipient bed, a further benefit of using a spacer during resection is that much smaller incisions are necessary for its removal, the debridement of the bony interface with endoscopic assistance, and the implantation of particulate grafts.

\section{Materials and Methods}

Two patients with ameloblastomas of the body of the mandible were treated as we have described previously. ${ }^{6}$ In each, we resected the tumour through an intraoral approach and placed a customised reconstruction plate, to which a silicone spacer had been secured with stainless steel wires (Fig. 1).

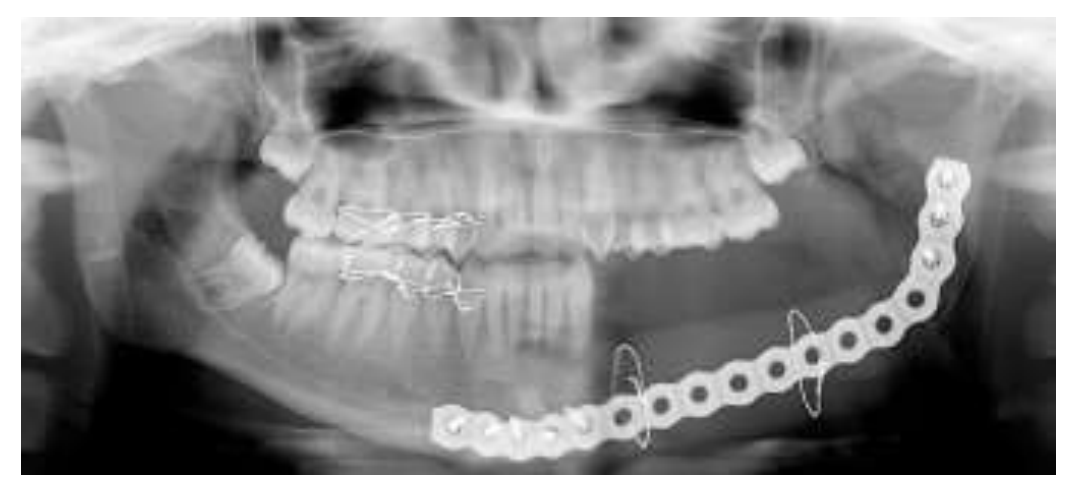

Fig. 1. Panoramic radiograph of the mandibular defect after resection of the tumour. The spacer is held in place with two stainless steel wires. 
Six weeks later, we used a $2 \mathrm{~cm}$ incision to access the spacer (in one patient the incision was retromandibular and in the other submental). We explored by blunt dissection until the securing wires were located and sectioned, and removed the spacer. We visualised the soft tissue bed and the extant mandibular interface using a rigid endoscope (Fig. 2) and debrided any adherant soft tissue.

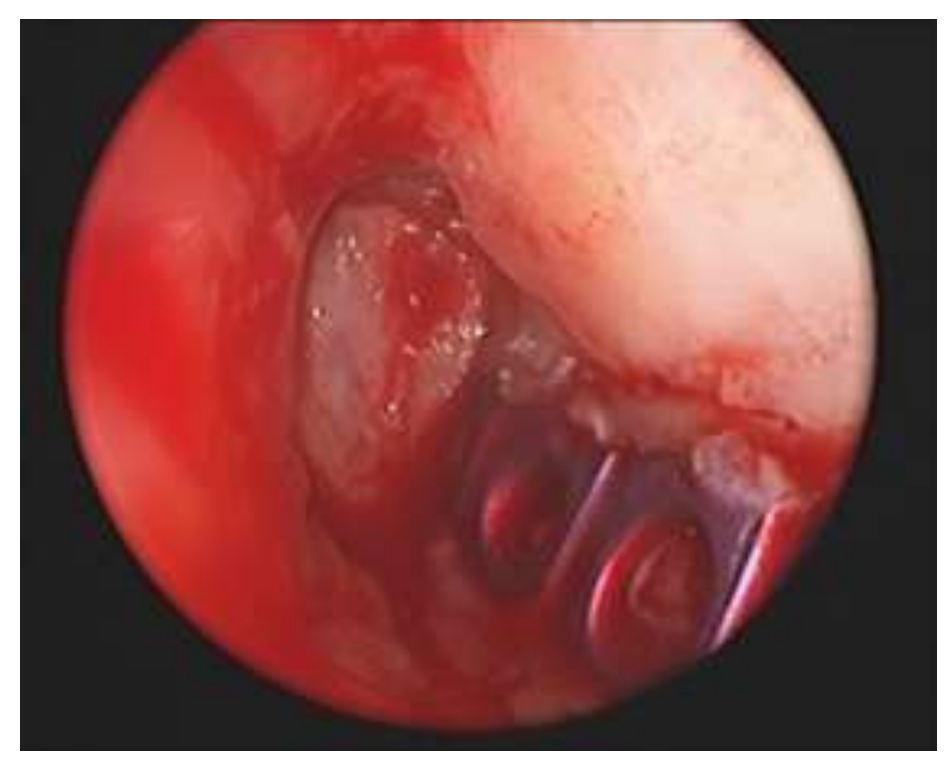

Fig. 2. Endoscopic view of the bony interface and reconstruction plate after removal of the spacer.

Bone harvested from the posterior iliac crest was milled in a power bone mill, transferred to 10 cc syringes and maximally compressed manually. We then removed the end of the syringe with a scalpel and introduced the bone through the access incisions into the space left behind. Initially we inserted small increments to the farthest interface and compressed them digitally to ensure good adaptation, and the space left was filled with the remaining bone graft. Maxillomandibular fixation was secured for six weeks. 


\section{Results}

Both patients healed uneventfully and the grafts matured well. Long-term radiographic follow up confirmed well-matured ossicles of adequate shape and height for the placement of implants, and prosthetic rehabilitation (Fig. 3). Extraoral scarring was reduced substantially (Fig. 4).

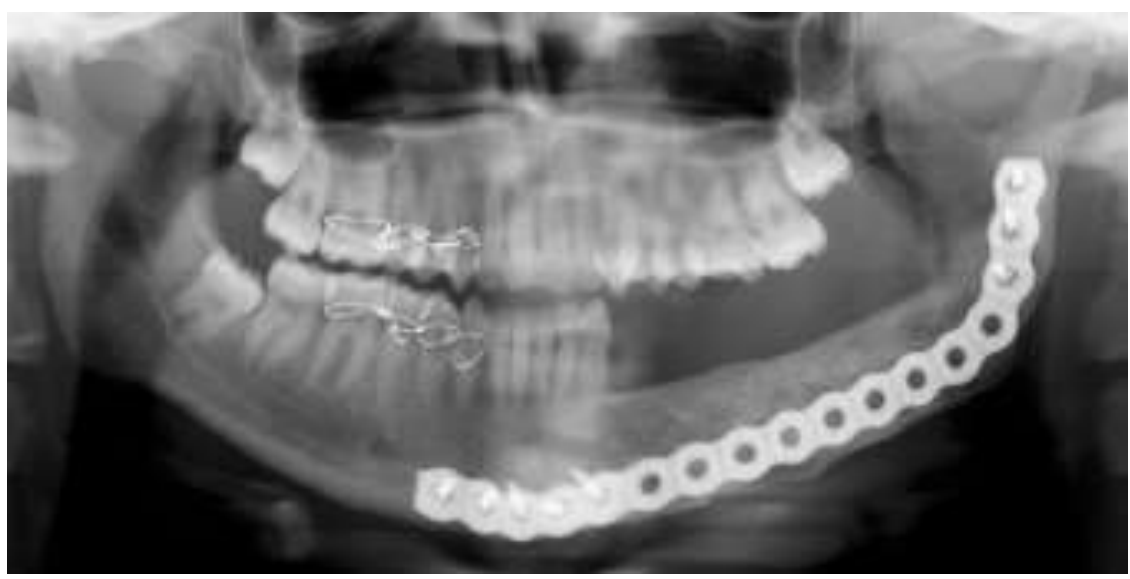

Fig. 3. Panoramic radiograph of the resultant ossicle that was implanted through the retromandibular approach.

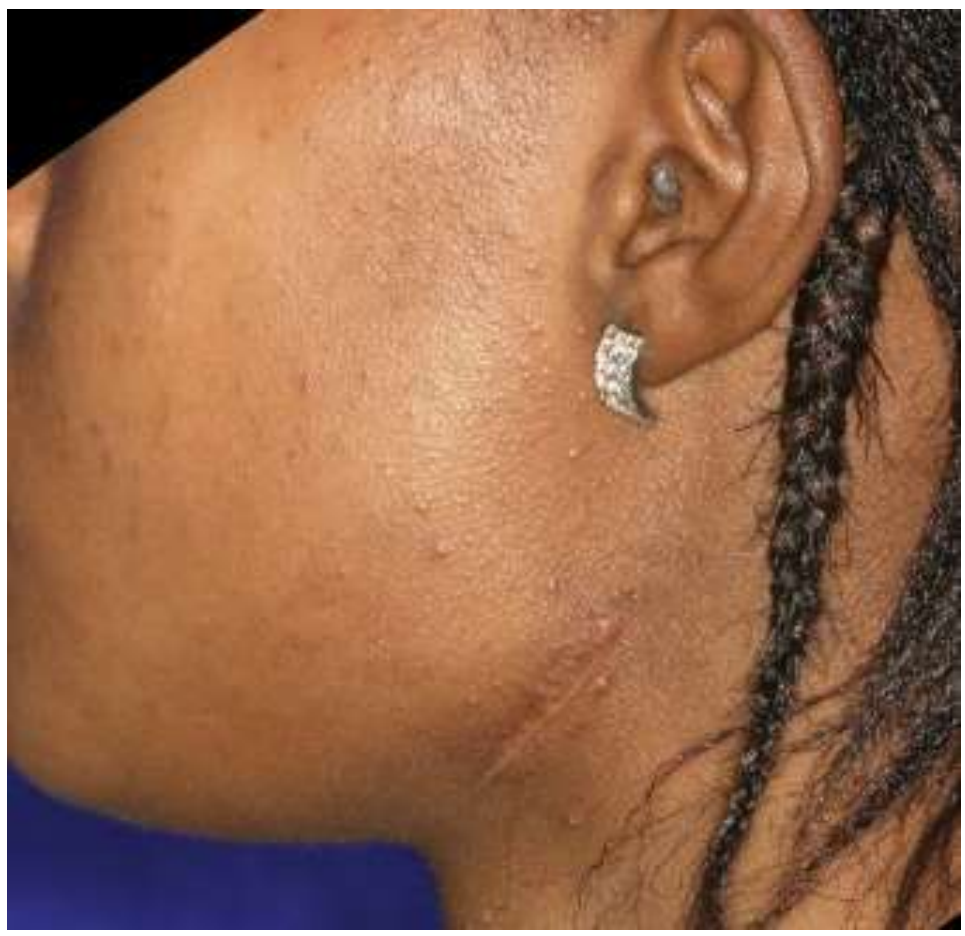

Fig. 4. The resultant scar after reconstruction of the mandible as shown in in Fig. 1, Fig. 2. 


\section{Discussion}

The use of an intermediate spacer for staged mandibular reconstruction facilitates implantation of a particulate bone graft. Moreover, it gives the surgeon the option of performing mandibular reconstruction through a much reduced incision without compromising the final outcome. The reduced incision expedites surgery, reduces the risk for iatrogenic injury to nerves and has significant cosmetic benefits. Due to the need for endoscopic access the technique may be limited to mandibular defects that do not cross the midline.

\section{References}

1. Boyne PJ, Zarem H. Osseous reconstruction of the resected mandible. Am J Surg. 1976 Jul; 132(1):49-53

2. Marx RE. Mandibular reconstruction. J Oral Maxillofac Surg. 1993 May;51(5):466-79

3. Iino M, Fukuda M, Nagai H, Hamada Y, Yamada H, Nakaoka K, Mori Y, Chikazu D, Saijo H, Seto I, Ohkubo K, Takato T. Evaluation of 15 mandibular reconstructions with Dumbach Titan MeshSystem and particulate cancellous bone and marrow harvested from bilateral posterior ilia. Oral Surg Oral Med Oral Pathol Oral Radiol Endod. 2009 Apr;107(4) :e1-8

4. Simon EN, Merkx MA, Kalyanyama BM, Shubi FM, Stoelinga PJ. Immediate reconstruction of the mandible after resection for aggressive odontogenic tumours: a cohort study Int J Oral Maxillofac Surg. 2013 Jan; 42(1):106-12.

5. Ferretti C, Muthray E, Rikhotso E, Reyneke J, Ripamonti U. Reconstruction of 56 mandibular defects with autologous compressed particulate corticocancellous bone grafts. Br J Oral Maxillofac Surg. 2016 Apr;54(3):322-6. doi: 10.1016/j.bjoms.2015.12.014. Epub 2016 Jan 21.

6. Ferretti C, Rikhotso E, Muthray E, Reyneke J. Interim reconstruction and space maintenance of mandibular continuity defects preceding definitive osseous reconstruction. Br J Oral Maxillofac Surg. 2013 Jun;51(4):319-25 